\title{
Comment on: Chlorpheniramine exerts anxiolytic-like effects and activates prefrontal 5-HT systems in mice (Miyata S. et al., Psychopharmacology, 2009)
}

\author{
Petros N. Karamanakos
}

Received: 23 October 2009 /Accepted: 18 December 2009/Published online: 19 January 2010

(C) Springer-Verlag 2010

\section{Dear Editor,}

I read with great interest the recently published article by Miyata et al. (2009) in Psychopharmacology, regarding the connection between the anxiolytic-like effect of chlorpheniramine (CPA) and the facilitation of serotonergic neurotransmission in the prefrontal cortex (PFC). Based on their results, Miyata et al. concluded that CPA has an anxiolyticlike effect in mice that is mediated, at least in part, by facilitation of serotonergic neurotransmission in the PFC, leaving, however, open the question of where is the exact site of action of CPA in the serotonergic system.

I believe that the authors of the above-mentioned article would have found some answers to the questions raised by their research if they had referred to a relatively recent publication of our laboratory team, regarding the effects of CPA on the brain serotonergic system of the rat (Karamanakos et al. 2004). According to the results of our paper, it was demonstrated for the first time that CPA acts as an agonist at the postsynaptic 5HT1A receptors, producing hyperlocomotion and hypothermia in rats. Pretreatment with the 5-HT1A receptor agonist 8-hydroxy- $N, N$-dipropyl-2-aminotetralin (8-OH-DPAT) or with the $5-\mathrm{HT} 1 \mathrm{~A} / \mathrm{B}$ receptor antagonist pindolol enhanced or blocked respectively these effects of CPA.

In view of our experimental results, it seems that the effects of CPA on the brain serotonergic system of the mice, as presented by Miyata et al., could be possibly attributed to the stimulation of 5-HT1A receptors. However, contrary to our results, Miyata et al. did not find any effect of CPA on the locomotor activity of the mice. In my opinion, this discrepancy derives from the fact that comparing to the high dose of CPA

\section{P. N. Karamanakos $(\bowtie)$}

Department of Neurosurgery, University Hospital of Kuopio,

Puijonlaaksontie 2,

70211 Kuopio, Finland

e-mail: petros.karamanakos@kuh.fi we administered to rats ( $40 \mathrm{mg} / \mathrm{kg}$, i.p.), which was enough to stimulate postsynaptic 5-HT1A receptors, Miyata et al. used low doses of CPA $(0.05-5 \mathrm{mg} / \mathrm{kg}$, i.p.), which probably stimulated presynaptic and not postsynaptic 5-HT1A receptors, the activation of which increases locomotor activity (Kalkman 1995). In support of this interpretation, Ahlenius et al. (1997) showed a dose-related biphasic pattern of effects of 8-OHDPAT on endurance performance of rats on a treadmill, with enhanced performance at a low dose and impaired performance at a high dose, attributed to stimulation of pre- and postsynaptic 5-HT1A receptors, respectively.

In conclusion, I suggest that Miyata et al. should have taken into consideration the results of our study, while explaining their own results, in order to strengthen their conclusions, giving at the same time a more accurate mechanistic explanation of the actions of CPA on the serotonergic system of the mice.

Yours sincerely

Petros N. Karamanakos, MD, Ph.D.

\section{References}

Ahlenius S, Kaur P, Salmi P (1997) Biphasic effects of 8-OH-DPAT on endurance of treadmill performance in the mail rat. Eur Neuropsychopharmacol 7:89-94

Kalkman HO (1995) RU 24969-induced locomotion in rats is mediated by 5 -HT1A receptors. Naunyn Schmiedebergs Arch Pharmacol 352:583-584

Karamanakos PN, Pappas P, Marselos M (2004) Involvement of the brain serotonergic system in the locomotor stimulant effects of chlorpheniramine in Wistar rats: implication of postsynaptic 5HT1A receptors. Behav Brain Res 148:199-208

Miyata S, Hirano S, Ohsawa M, Kamei J (2009) Chlorpheniramine exerts anxiolytic-like effects and activates prefrontal 5-HT systems in mice. Psychopharmacology. doi:10.1007/s00213-009-1695-0 